\title{
Elabela prevents angiotensin II-induced apoptosis and inflammation in rat aortic adventitial fibroblasts via the activation of FGF21-ACE2 signaling
}

\author{
Juan-Juan Song ${ }^{1} \cdot$ Mei Yang ${ }^{1} \cdot$ Ying Liu $^{1} \cdot$ Jia-Wei Song ${ }^{1} \cdot$ Xiao-Yan Liu $^{1,2} \cdot$ Ran Miao $^{2} \cdot$ Zhen-Zhou Zhang $^{1,3} \cdot$ Yu Liu ${ }^{1,3}$. \\ Yi-Fan Fan ${ }^{1} \cdot$ Qian Zhang ${ }^{1} \cdot$ Ying Dong ${ }^{1} \cdot$ Xin-Chun Yang ${ }^{1} \cdot$ Jiu-Chang Zhong ${ }^{1,2,3}$
}

Received: 23 June 2020 / Accepted: 10 August 2021 / Published online: 28 August 2021

(c) The Author(s), under exclusive licence to Springer Nature B.V. 2021

\begin{abstract}
Apoptosis, inflammation, and fibrosis contribute to vascular remodeling and injury. Elabela (ELA) serves as a crucial regulator to maintain vascular function and has been implicated in the pathogenesis of hypertensive vascular remodeling. This study aims to explore regulatory roles and underlying mechanisms of ELA in rat aortic adventitial fibroblasts (AFs) in response to angiotensin II (ATII). In cultured AFs, exposure to ATII resulted in marked decreases in mRNA and protein levels of ELA, fibroblast growth factor 21 (FGF21), and angiotensin-converting enzyme 2 (ACE2) as well as increases in apoptosis, inflammation, oxidative stress, and cellular migration, which were partially blocked by the exogenous replenishment of ELA and recombinant FGF21, respectively. Moreover, treatment with ELA strikingly reversed ATII-mediated the loss of FGF21 and ACE2 levels in rat aortic AFs. FGF21 knockdown with small interfering RNA (siRNA) significantly counterbalanced protective effects of ELA on ATII-mediated the promotion of cell migration, apoptosis, inflammatory, and oxidative injury in rat aortic AFs. More importantly, pretreatment with recombinant FGF21 strikingly inhibited ATII-mediated the loss of ACE2 and the augmentation of cell apoptosis, oxidative stress, and inflammatory injury in rat aortic AFs, which were partially prevented by the knockdown of ACE2 with siRNA. In summary, ELA exerts its anti-apoptotic, anti-inflammatory, and antioxidant effects in rat aortic AFs via activation of the FGF21-ACE2 signaling. ELA may represent a potential candidate to predict vascular damage and targeting the FGF21-ACE2 signaling may be a promising therapeutic intervention for vascular adventitial remodeling and related disorders.
\end{abstract}

Keywords Elabela $\cdot$ Fibroblast growth factor $21 \cdot$ Angiotensin-converting enzyme $2 \cdot$ Apoptosis $\cdot$ Inflammation $\cdot$ Adventitial fibroblasts

\section{Introduction}

The adventitial remodeling of arteries may initially be adaptive, but eventually, it becomes maladaptive and compromises organ function, contributing to cardiovascular

Jiu-Chang Zhong

jczhong@sina.com

1 Heart Center and Beijing Key Laboratory of Hypertension, Beijing Chaoyang Hospital, Capital Medical University, Beijing 100020, China

2 Medical Research Center, Beijing Chaoyang Hospital, Capital Medical University, Beijing 100020, China

3 Department of Cardiology, Beijing Chaoyang Hospital, Capital Medical University, Beijing 100020, China complications of hypertension (Intengan and Schiffrin 2001). Remodeled vessels are characterized by adventitial thickening and increased number of fibroblasts and phenotypic change of adventitial fibroblasts (AFs) to myofibroblasts. AFs function as the most abundant cell type in the adventitia and appear to be a sensor of vascular wall disruption and dysfunction as well as an early responder and activator of the blood vessel in response to injury or stress (Intengan and Schiffrin 2001; Wang et al. 2014a). Indeed, emerging evidence has been accumulated for the pivotal significance of abnormal migration, proliferation, and excessive inflammatory response of AFs in the initiation, progression, and exacerbation of vascular remodeling (Chai et al. 2018).

Elabela (ELA), a novel endogenous ligand of apelin receptor (APJ), is recognized for its various roles in vasodilatation, vessel development, differentiation, and 
cardiovascular function in adult mammalian systems (Ma et al. 2020). The ELA-APJ system has been implicated to prevent pressure overload-induced hypertension and cardiovascular dysfunction via the suppression of angiotensinconverting enzyme (ACE) expression and pathogenic angiotensin II (ATII) signaling as well as the promotion of the level of ACE2 (Ma et al. 2020; Sato et al. 2017; Murza et al. 2016; Yang et al. 2017). Additionally, ELA serves as a chemoattractant for the angioblasts and regulates this medial migration, which is crucial for normal vasculature development during embryogenesis (Helker et al. 2015). The ELAAPJ signaling stimulates angiogenesis of human umbilical vascular endothelial cells and induces relaxation of mouse aortic blood vessels (Wang et al. 2015). Fibroblast growth factor 21 (FGF21) is a key component in hormone-mediated multi-organ crosstalk among adipose tissue, liver, pancreas, blood vessels as well as heart and regulates the homeostasis of lipid, glucose, and energy metabolism by activating FGF receptor and its cofactor $\beta$-Klotho (Luo et al. 2018). Clinical studies have unraveled the strong positive correlation of FGF21 with a panel of cardiovascular diseases. Increasing evidence point out that FGF21 is an endogenous cardiovascular protective peptide to exert vascular protective effects via the induction of adiponectin indirectly (Lin et al. 2015; Yan et al. 2015). Therefore, FGF21 is known as a biomarker and potential regulator to predict or prevent vascular disease incidence. However, the regulatory roles of ELA in FGF21 signaling in the vessels remain largely unknown.

Intriguingly, ACE2 is recently discovered as a co-receptor for the severe acute respiratory syndrome coronavirus 2 (SARS-CoV2) entry for the novel coronavirus 2019 pneumonia and has been shown to prevent cardiovascular fibrosis and dysfunction by counterbalancing the renin-angiotensin system (RAS) (Gheblawi et al. 2020). ATII, a main member of the RAS, induces the injury and dysfunction of endothelial cells by enhancing oxidative stress, adhesion molecules, and inflammatory cytokines in the vessel wall, which were abolished by recombinant human ACE2 (Ma et al. 2020; Gheblawi et al. 2020; Agarwal 2003; Williams and Scholey 2018). Moreover, ACE2 activation ameliorates endothelial dysfunction in rats with pulmonary arterial hypertension through mediating phosphorylation of endothelial nitric oxide synthase, highlighting the potential importance of ACE2 in the regulation of vascular function in pathologic conditions ( $\mathrm{Li}$ et al. 2017). In our and other research groups have demonstrated that the activation of ACE2 signaling has been shown to mediate the beneficial effects of FGF21 on ATII-induced hypertension and vascular injury (Pan et al. 2018). However, the exact role of the FGF21-ACE2 axis in vascular adventitia is far from clear. Thus, we evaluate the regulatory roles and underlying mechanisms of ELA in rat aortic AFs in response to ATII.

\section{Materials and methods}

\section{Primary culture of rat aortic AFs}

The protocol of the study was approved by the Animal Research Ethics Committee at Beijing Chaoyang Hospital Affiliated to Capital Medical University. Primary rat aortic AFs were obtained from ascending aortas of 5- or 6-week-old male Sprague-Dawley rats [Charles River, Beijing, China, License Number: SCXK (Beijing) 2016-0006] as previously described (Xu et al. 2016). Briefly, thoracic aortas were dissected and placed in Dulbecco's modified Eagle's medium (DMEM). Then, the medial and intimal layers of the aortas were separated from the adventitia under sterile conditions. The isolated adventitia was cut into $1 \mathrm{~mm}^{3}$ segments and placed in DMEM supplemented with $20 \%$ heat-inactivated fetal bovine serum (FBS), $1 \%$ L-glutamine, $100 \mathrm{U} / \mathrm{mL}$ penicillin, and $100 \mathrm{mg} / \mathrm{mL}$ streptomycin. The explants were cultured for 10-14 days for passage in a humidified atmosphere gassed with $5 \% \mathrm{CO}_{2}$. The rat aortic AFs from 3rd to 6th generations were used in the subsequent experiments and incubated in a serum-free medium for $24 \mathrm{~h}$ before different stimulation. Recombinant human ELA-32 (100 nM), recombinant FGF21 (50 nM) were added to AFs for $1 \mathrm{~h}$ before $24 \mathrm{~h}$ exposure of ATII $(100 \mathrm{nM})$, respectively.

\section{Cell transfection with siRNA}

For transient transfection, rat aortic $\mathrm{AFs}$ were transfected at $70 \%$ confluence in 6-well plates with $50 \mathrm{nM}$ small interfering RNAs (siRNAs) targeting ELA-32, FGF21, and ACE2 (GenePharma, Shanghai, China) and with corresponding negative control (NC) siRNAs, respectively, using Lipofectamine 3000 (Invitrogen, CA, USA) according to the manufacturer's protocols. After $6-8 \mathrm{~h}$ of transfection, the medium was changed to fresh DMEM supplemented with $10 \%$ FBS and cells were maintained until $48-72 \mathrm{~h}$ before further experiments. To determine the knockdown efficiency, polymerase chain reaction (PCR) and immunoblotting analysis were performed to confirm the gene downregulation. The following siRNA sequences were used: ELA siRNA: sense 5'-CUUCGAUAAGAAAGU AAUAGA-3' antisense 5'-UAUUACUUUCUUAUCGAAGAA-3', FGF21 siRNA: sense 5'-CGACAGAGGUAU CUCUACACA-3' antisense 5'-UGUAGAGAUACCUCU GUCGGA-3', ACE2 siRNA: sense 5'-CGAAGUUGAUGGAAGUGUACC- $3^{\prime}$ antisense 5'-UACACUUCCAUC AACUUCGUU- ${ }^{\prime}$. NC siRNA: sense 5'-UUCUCCGAA CGUGUCACGUTT-3' antisense 5'-ACGUGACAC-GUU CGGAGAATT-3'. 


\section{Wound-healing assay and dihydroethidium staining}

Rat aortic AFs were cultured in 6-well plates at a density of $1 \times 10^{5}$ cells/well. A scratch was gently made at the cell surface using a sterile pipette tip ( $1 \mathrm{~mL}$ ) after growth to $70 \%$ confluence. At 0 and $24 \mathrm{~h}$ after wounding, wound width was measured under a microscope. The cell migration capability was expressed by the relative migration index with Image $\mathrm{J}$ software. The production of reactive oxygen species (ROS) was measured by the dihydroethidium (DHE) staining method. Briefly, AFs pretreated were incubated with DHE $(10 \mu \mathrm{M})$ at $37^{\circ} \mathrm{C}$ for $30 \mathrm{~min}$ in the dark. Then, cells were washed thrice with phosphate buffer saline (PBS) to remove the redundant DHE. Fluorescence intensity was determined using the Olympus IX51 microscope (Olympus, Tokyo) to indicate the generation of ROS.

\section{Flow cytometry array}

Cell apoptosis detection was performed with Annexin V-FITC and propidium iodide (PI) apoptosis detection kit (BD Pharmingen, USA) according to the manufacturer's instruction. Briefly, pre-treated AFs were harvested and washed with PBS, followed by suspension in $100 \mu \mathrm{L}$ binding buffer containing $5 \mu \mathrm{L}$ Annexin V-FITC for $15 \mathrm{~min}$ at $37^{\circ} \mathrm{C}$ in the dark. Then $5 \mu \mathrm{L}$ PI was added for a further $5 \mathrm{~min}$ incubation. For each sample, 10,000 cells were analyzed with an Accuri ${ }^{\mathrm{TM}}$ C6 flow cytometer (BD Pharmingen, San Diego, CA, USA).

\section{Western blot}

Standard western blot protocols were followed to examine the protein levels in rat aortic AFs as previously described (Xu et al. 2016). Total protein was extracted from AFs with radio immune-precipitation assay (RIPA) buffer (Beyotime, Beijing, China) supplemented with protease inhibitor for 30 min on the ice. A bicinchoninic acid protein assay kit was used to measure protein concentration. Subsequently, proteins were transferred to PVDF membrane (Millipore, USA), blocked with $5 \%$ non-fat milk for $2 \mathrm{~h}$ and incubated overnight at $4{ }^{\circ} \mathrm{C}$ with primary antibodies against Bax (\#2772), Bcl-2 (Sc-7382), ACE2 (ab108252), FGF21 (ab171941), GAPDH (\#2118). Then, the membranes were incubated with a secondary antibody and detected using the Odyssey infrared imaging system (LI-COR, Lincoln, NE, USA). Antibodies were obtained from Cell Signaling Technology (Beverly, MA), Santa Cruz Biotechnology (Santa Cruz, CA), and Abcam Inc (Cambridge, MA), respectively. GAPDH was used as an endogenous control. The intensity of blots was analyzed by Image J software.

\section{Reverse transcription PCR (RT-PCR)}

Total RNA was extracted from rat aortic AFs with TRIzol reagent (Invitrogen, CA, USA) and was converted to cDNA using PrimeScript RT reagent kit (Takara, Tokyo, Japan). SYBR quantitative RT-PCR kit (Takara, Tokyo, Japan) was used and amplified with ABI Prism 7500 Sequence Detection System (Applied, Biosystems, CA). Amplification conditions were set as a 40-cycle program $\left(95{ }^{\circ} \mathrm{C}\right.$ for $5 \mathrm{~s}, 60{ }^{\circ} \mathrm{C}$ for $34 \mathrm{~s}$ ). The mRNA levels of ELA32, FGF21, ACE2, interleukin-1 $\beta$ (IL-1 $\beta$ ), IL-6, tumor necrosis factor- $\alpha$ (TNF- $\alpha)$, and monocyte chemoattractant protein-1 (MCP-1) were examined and analyzed. GAPDH was used as an endogenous control. The following primers were used: ELA: forward 5'-GTCACTGATCTCCTT GGTTA-3' reverse 5'-TGCTCTAGTCTAGTCTGTCT-3', ACE2: forward 5'-CTTACGAGCCTC-CTGTCACC-3' reverse 5'-ATGCCAACCACTACCGTTCC-3', FGF21: forward 5'-GAGATCAGGGAGGACGGAAC-3' reverse 5'-CCTAGAGGCTTTGACACCCG-3', IL-1 $\beta$ : forward 5'-GACTTCACCATGGAACCCGT-3' reverse 5'-GGA GACTGCC-CATTCTCGAC-3', IL-6: forward 5'-GAG ACTTCCAGCCAGTTGCC-3' reverse 5'-TGAAGTCTC CTCTCCGGACTT-3', TNF- $\alpha$ : forward 5'-ATTGTGGCT CTGGGT-CCAAC-3' reverse 5'-AGCGTCTCGTGTGTT TCTGA-3' MCP-1: forward 5'-CTTC-CTCCACCACTAT GCAGG-3' reverse 5'-GATGCTACAGGCAGCAACTG-3', GAPDH: forward 5'-AGTGCCAGCCTCGTCTCATA-3' reverse 5'-TGAACTTGCC-GTGGGTAGAG-3'. All samples were run at least in triplicates.

\section{Immunological assay}

Cell supernatants of rat aortic AFs culture medium were collected and FGF21 concentrations in the culture medium were measured by using a rat-specific FGF21 SampleStep ELISA Kit (Abcam, Cambridge, UK, ab223589) according to the manufacturer's protocols.

\section{Statistical analyses}

GraphPad Prism version 8.0.1 was used to draw histograms. Statistical analysis was performed with IBM SPSS Statistics 24 software. Data are presented as mean \pm SD. Significant differences between and within multiple groups were examined using ANOVA for repeated measures, followed by Duncan's multiple range test. The Student's t-test (for unpaired samples) was performed to compare the data between two groups. $P<0.05$ was considered statistically significant. 


\section{Results}

\section{Treatment with ELA alleviated ATII-induced cell apoptosis and oxidative stress in rat aortic AFs}

Firstly, we evaluated the regulatory roles of ELA in cell apoptosis and ROS generation in rat aortic AFs. Compared to the control group, exposure to ATII induced a remarkable reduction in the mRNA level of ELA in rat aortic AFs, which was rescued by exogenous replenishment of ELA (Fig. 1a). Meanwhile, gene silencing of ELA with siRNA decreased the mRNA expression of ELA in rat aortic AFs in response to ATII compared with the negative control group (Fig. 1a). These findings were consistent with the previous report that exhibited a declined circulating level of ELA in essential hypertension associated with vascular
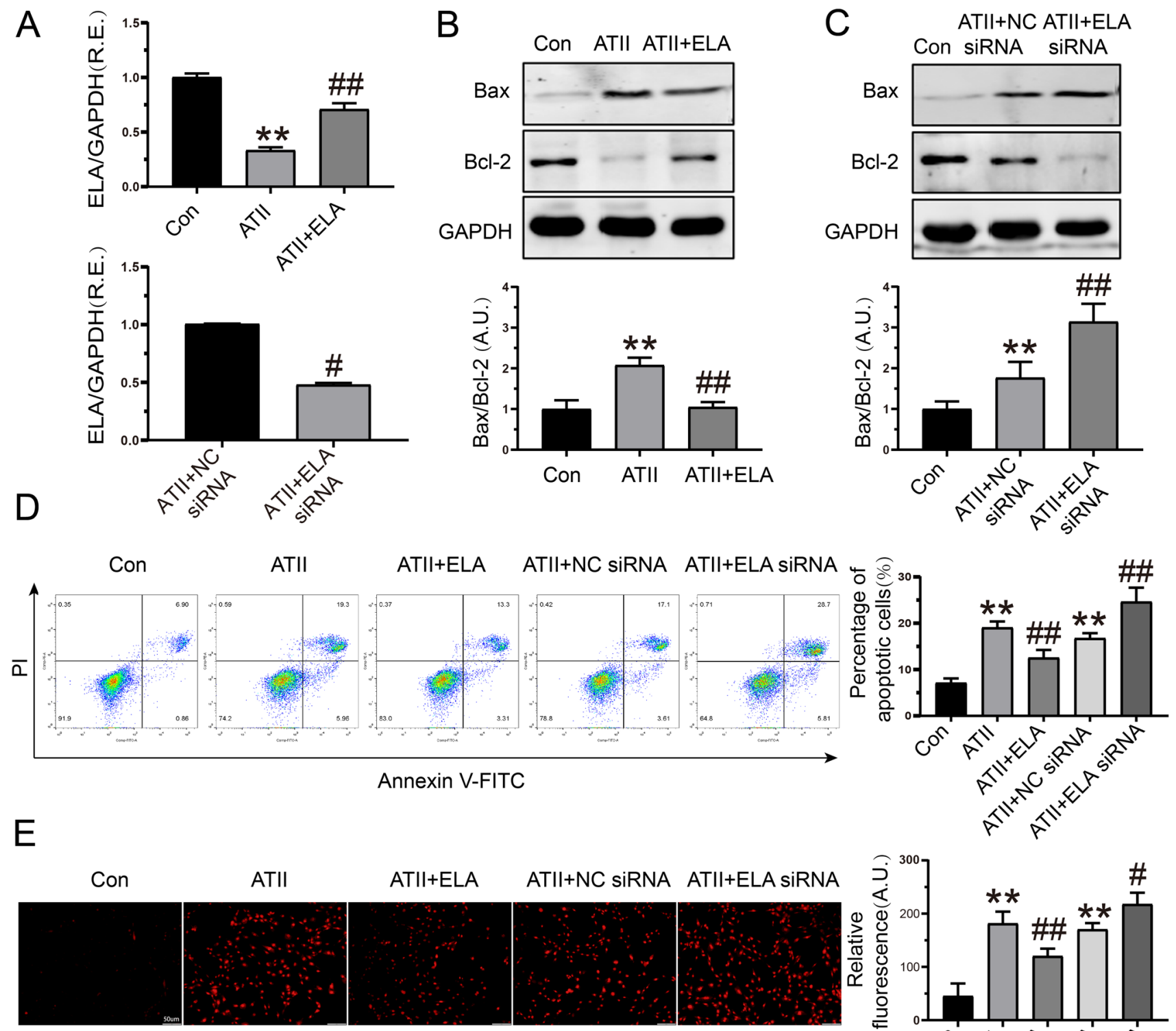

Annexin V-FITC

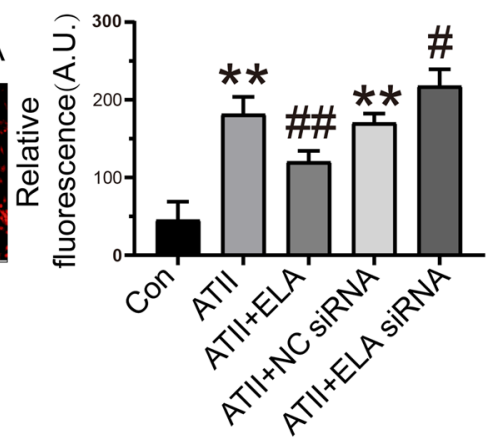

Fig. 1 Regulatory roles of ELA in cell apoptosis and oxidative stress in rat aortic AFs. a Relative mRNA levels of ELA in rat aortic AFs in response to ATII, ELA, and ELA siRNA. b, c Representative Western blots images and quantification to detect protein levels of bax and bcl-2 in rat aortic AFs. d The detection of cell apoptosis by flow cytometry array with quantification in rat aortic AFs. e Dihydroethidium staining to measure ROS generation in rat aortic AFs. GAPDH was used as an endogenous control. $n=3-4$ for each group except for a where $\mathrm{n}=6$. $* * P<0.01$ compared with the control group; $\# P<0.05, \# \# P<0.01$ compared with ATII or ATII + NC siRNA group. A.U. arbitrary units, R.E. relative expression, $A F s$ adventitial fibroblasts, ATII angiotensin II, NC negative control, ELA elabela, $R O S$ reactive oxygen species 

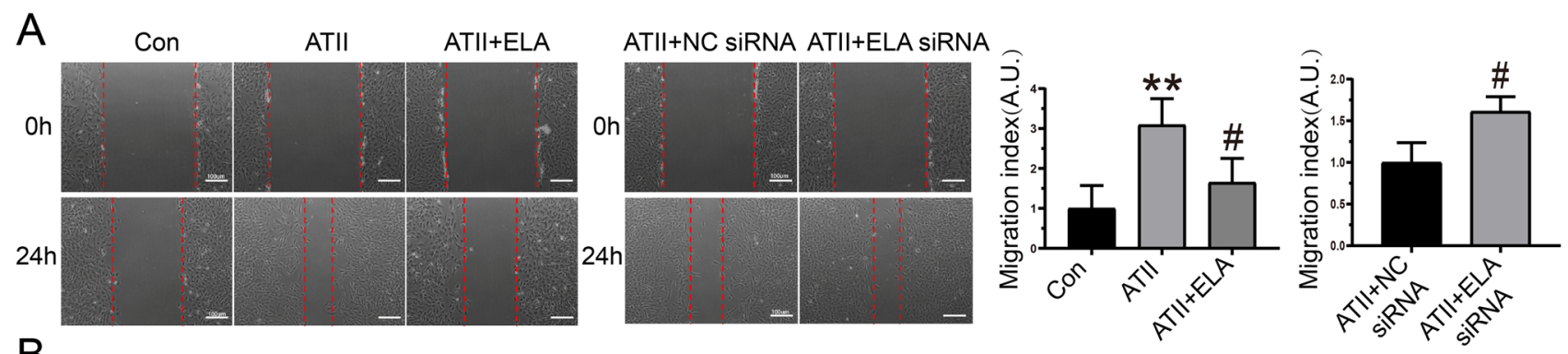

B
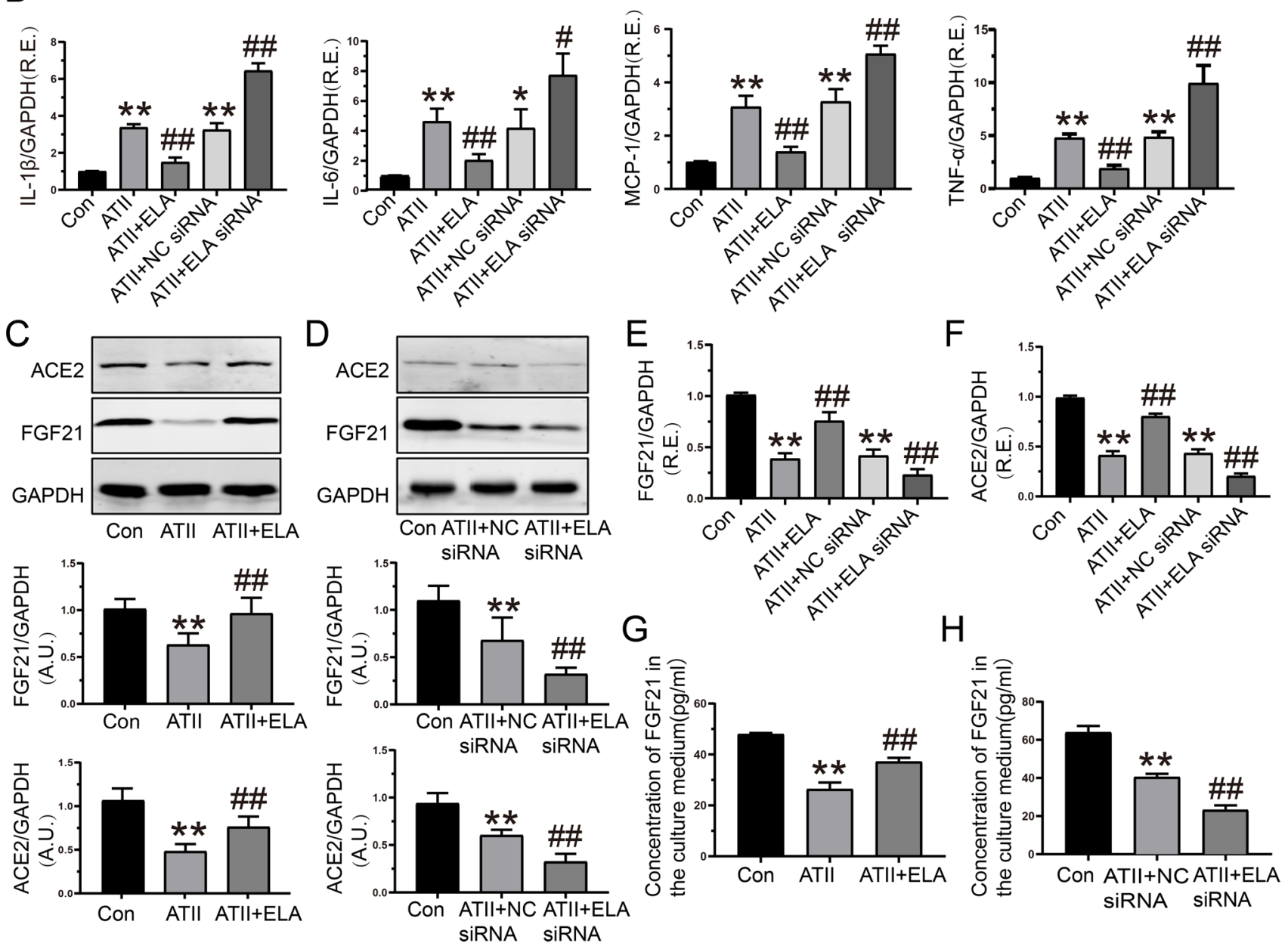

Fig. 2 Effects of ELA on cell migration and inflammatory response in rat aortic AFs in response to ATII. a Representative wound-healing images and quantification at 0 and $24 \mathrm{~h}$ in rat aortic AFs. b Relative mRNA levels of IL- $1 \beta$, IL-6, MCP-1, and TNF- $\alpha$ in rat aortic AFs. c, $\mathbf{d}$ Protein levels of ACE2 and FGF21 in rat aortic AFs pretreated with ELA and ELA siRNA by Western blot analysis. e, f The mRNA levels of FGF21 and ACE2 were elevated by ELA stimulation but were reduced by ELA knockdown, respectively. $\mathbf{g}, \mathbf{h}$ The concentration of FGF21 in the cell culture medium was measured in the presence of ATII, ELA, and ELA siRNA by

ELISA kit. GAPDH was used as an endogenous control. $n=3-6$ for each group. $* P<0.05, * * P<0.01$ compared with the control group; $\# P<0.05$, $\# P<0.01$ compared with ATII or ATII+NC siRNA group. A.U. arbitrary units, R.E. relative expression, AFs adventitial fibroblasts, $A T I I$ angiotensin II, $N C$ negative control, ELA elabela, $F G F 21$ fibroblast growth factor 21, $A C E 2$ angiotensin-converting enzyme $2, I L-1 \beta$ interleukin- $1 \beta, I L-6$ interleukin-6, TNF- $\alpha$ tumor necrosis factor- $\alpha, M C P-1$, monocyte chemoattractant protein-1 


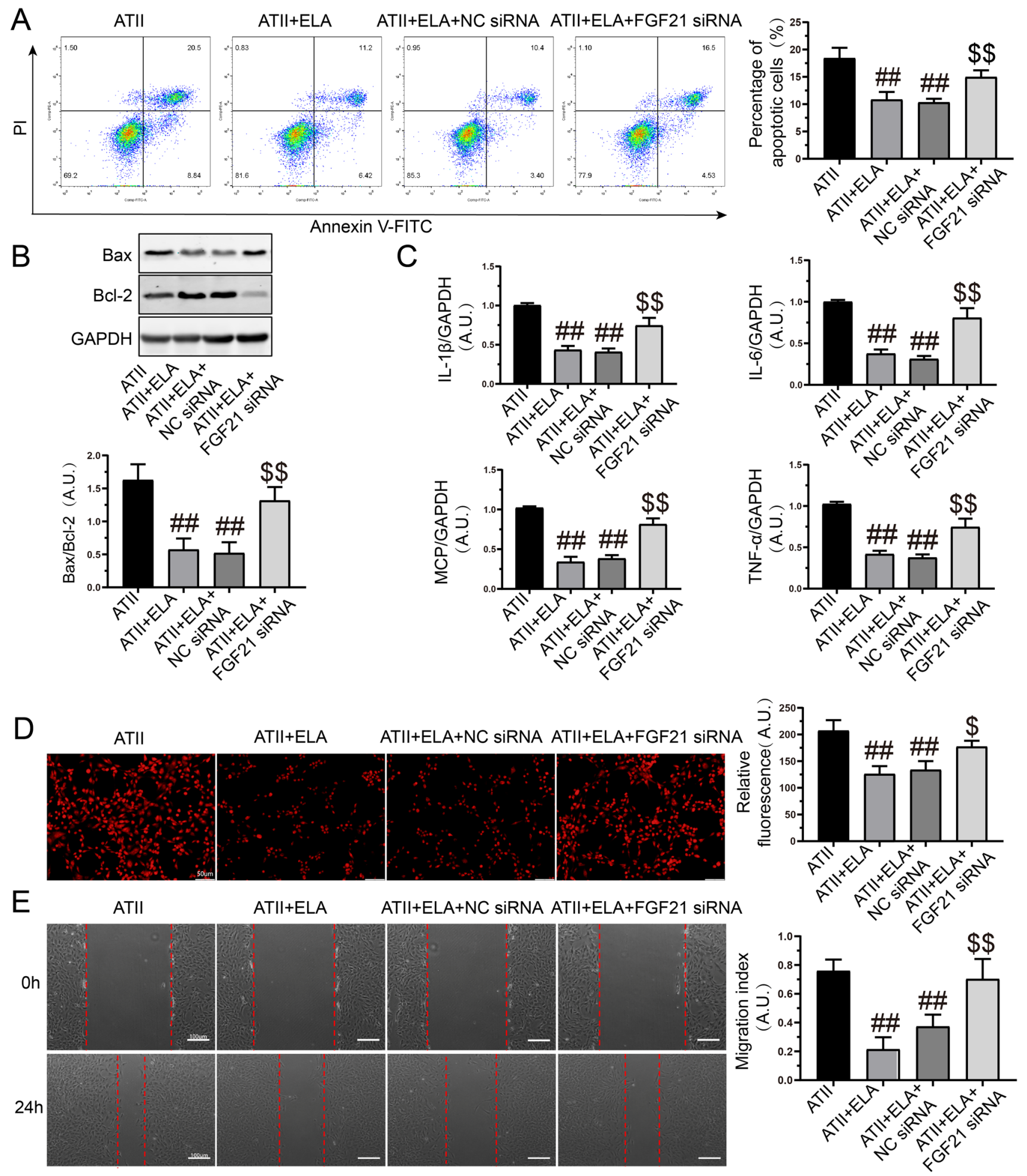

injury and dysfunction ( $\mathrm{Li}$ et al. 2020). The bax/bcl-2 ratio was elevated after ATII stimulation in rat aortic AFs, which was prevented by pretreatment with ELA while was further enhanced by knockdown of ELA with siRNA (Fig. 1b, c). Moreover, the cell apoptosis detected with flow cytometer was significantly increased in rat aortic AFs in response to ATII, which was reversed by exogenous replenishment of ELA (Fig. 1d). Notably, compared to the negative control group, ELA knockdown contributed to a higher cell apoptosis rate in rat aortic AFs (Fig. 1d). As expected, DHE fluorescence images depicted a significant increase in the 
4Fig. 3 FGF21 knockdown prevented the protective effects of ELA on ATII-mediated promtion of apoptosis, inflammation, oxidative stress, and cell migration in rat aortic AFs. a Flow cytometry to detect cell apoptosis in rat aortic AFs in the presence of ATII, ELA, and FGF21 siRNA. b Representative Western blots images and qualification to measure the protein levels of bax and bcl-2 in rat aortic AFs. $\mathbf{c}$ Relative mRNA levels of IL- $1 \beta$, IL-6, MCP-1, and TNF- $\alpha$ in rat aortic AFs by RT-PCR. d, e The level of ROS and cell migration was detected in rat aortic AFs by dihydroethidium staining and wound healing analysis, respectively. GAPDH was used as an endogenous control. $\mathrm{n}=3-4$ for each group except for $\mathbf{c}$ where $\mathrm{n}=5-6$. \#\#P<0.01 compared with the ATII group. $\$ \mathrm{P}<0.05, \$ \$ P<0.01$ compared with $\mathrm{ATII}+\mathrm{ELA}+\mathrm{NC}$ siRNA group; $A . U$. arbitrary units, R.E. relative expression, $A F s$ adventitial fibroblasts, $A T I I$ angiotensin II, $N C$, negative control, ELA elabela; $I L-1 \beta$, interleukin- $1 \beta, I L-6$, interleukin-6, $T N F-\alpha$ tumor necrosis factor- $\alpha, M C P-1$, monocyte chemoattractant protein-1, ROS, reactive oxygen species

superoxide production in rat aortic AFs pre-treated with ATII, which was further exacerbated by ELA siRNA but was suppressed by the administration of ELA (Fig. 1e). Collectively, these results indicated the protective effects of ELA on ATII-induced apoptotic and oxidative injury in rat aortic AFs.

\section{ELA knockdown exacerbated ATII-induced cell migration and inflammatory injury in rat aortic AFs}

To explore the influence of ELA on cell migration and inflammatory injury, we performed a wound-healing array and RT-PCR in rat aortic AFs in response to ATII, respectively. As expected, exposure to ATII resulted in significant increases in cell migration and inflammatory cytokines levels in rat aortic AFs, which were further augmented by ELA knockdown with siRNA (Fig. 2a, b). Nevertheless, ATIImediated pro-migratory and pro-inflammatory effects were prevented by ELA stimulation in rat aortic AFs (Fig. 2a, b). The mRNA levels of proinflammatory cytokines including IL-1 $\beta$, IL-6, MCP-1, and TNF- $\alpha$ were strikingly enhanced in rat aortic AFs after ATII treatment, which was further amplified by ELA siRNA but was strikingly blocked by the replenishment of ELA (Fig. 2b). These results suggested that gene silencing of ELA exacerbated ATII-induced cell migration and inflammatory injury in rat aortic AFs.

\section{Treatment with ELA activated the FGF21/ACE2 signaling in rat aortic AFs}

FGF21 has been shown to promote angiogenesis in microvascular endothelial cells and alleviate endothelial dysfunction and vascular injury (Huang et al. 2019; Ying et al. 2019). However, little is known about the role of FGF21 in vascular adventitial cells. Since the exact protective roles of ELA in rat aortic AFs, we further investigated the inner relationship between ELA and FGF21 in rat AFs in response to ATII. Protein levels of FGF21 and ACE2 were reduced in rat aortic AFs exposed to ATII, which was rescued by the supplement of ELA (Fig. 2c). These results were further verified by real-time quantitative RT-PCR analysis (Fig. 2e, f). Otherwise, compared to the negative control group, the magnitude of the decline in protein and mRNA levels of FGF21 and ACE2 was lower after gene silencing of ELA with siRNA (Fig. 2d-f). Furthermore, we also explore the concentration of FGF21 in AFs cell culture medium. ATII stimulation induced a decline in the concentration of FGF21 in the cell culture medium of rat aortic AFs, which was rescued by the treatment with ELA but was further reduced by ELA siRNA (Fig. 2g, h). Thus, these data suggested that the activation of FGF21/ACE2 signaling is partially responsible for the protective effects of ELA against ATII-induced cellular damage and dysfunction in rat aortic AFs.

\section{FGF21 knockdown blunted the protective effects of ELA on ATII-induced cellular injury and dysfunction in rat aortic AFs}

Next, we further investigated whether FGF21 knockdown would affect the protective actions of ELA in rat aortic AFs in response to ATII. Consistently, the replenishment of ELA prevented the AII-mediated increases in cell apoptosis (Fig. 3a, b), inflammatory response (Fig. 3c), oxidative stress (Fig. 3d), and cell migration (Fig. 3e) in rat aortic AFs, which were reversed by the transfection of FGF21 siRNA. Importantly, the ATII-mediated upregulation of bax/bcl-2 ratio and elevated mRNA levels of proinflammatory cytokines including IL- $1 \beta$, IL- 6 , MCP- 1 , and TNF- $\alpha$ were inhibited with ELA treatment in rat aortic AFs (Fig. 3b, c). Moreover, FGF21 knockdown significantly reversed the protective impacts of ELA on ATII-induced actions (Fig. 3a-e), indicating the beneficial effects of ELA against ATII-induced cellular injury and dysfunction in rat aortic AFs by activating the FGF21 signaling.

\section{FGF21 ameliorated ATII-induced the accumulation of apoptosis, oxidative stress, cell migration, and inflammatory response in rat aortic AFs}

To evaluate the influence of FGF21 on ATII-mediated actions, we examined whether recombinant FGF21 could affect vascular injury. Intriguingly, FGF21 knockdown with siRNA further deteriorated ATII-mediated pro-apoptotic (Fig. 4a, c), pro-oxidant (Fig. 4d), pro-migratory (Fig. 4e), and pro-inflammatory (Fig. 4f) effects in rat aortic AFs, which were rescued by recombinant FGF21 (Fig. 4a, b, d-f). ATII stimulation induced significant increases in cell apoptosis and bax/bcl-2 ratio, which were further accelerated by FGF21 knockdown but were suppressed with FGF21 treatment (Fig. 4a-c). Additionally, exposure to ATII promoted 

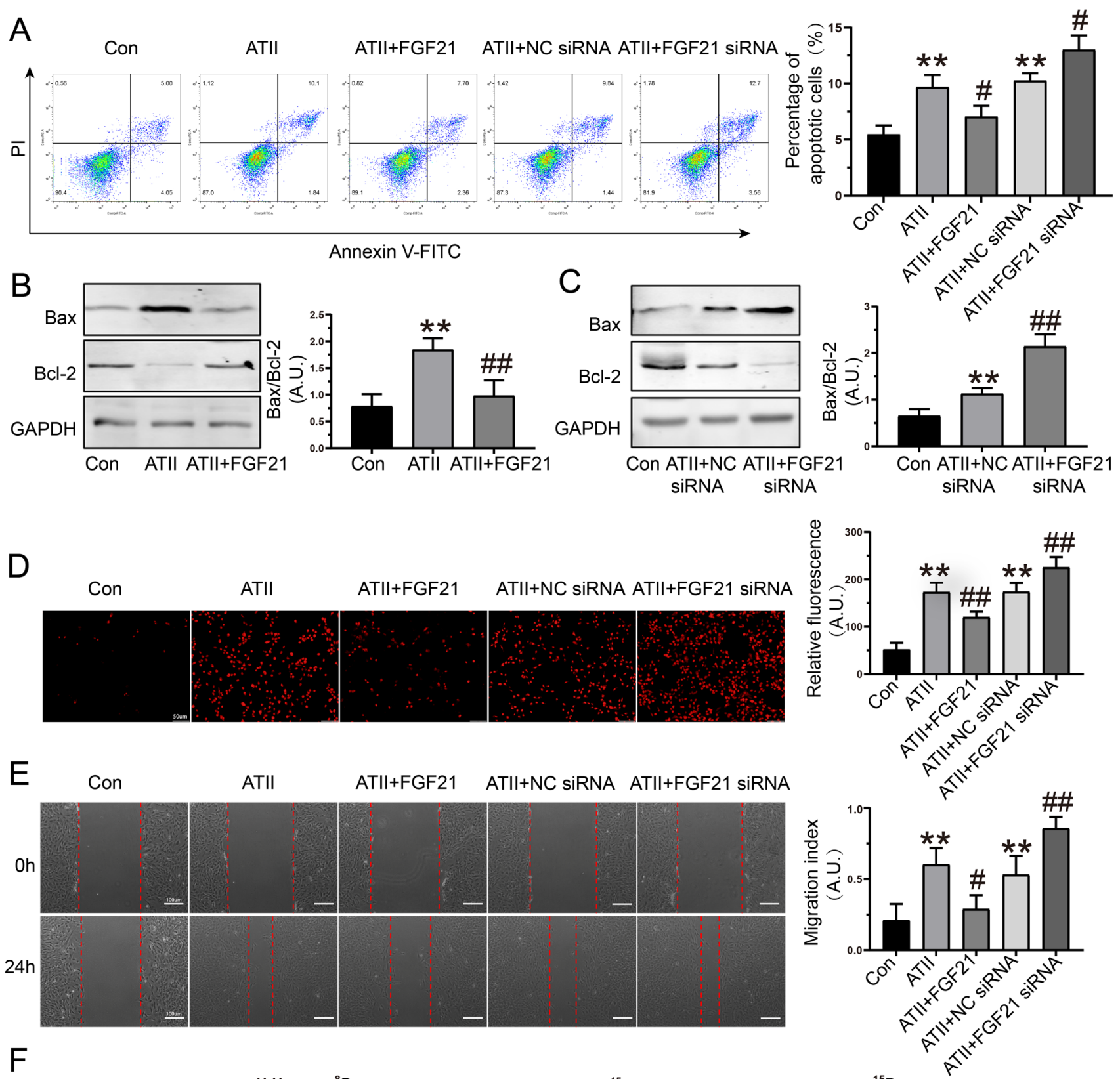

F
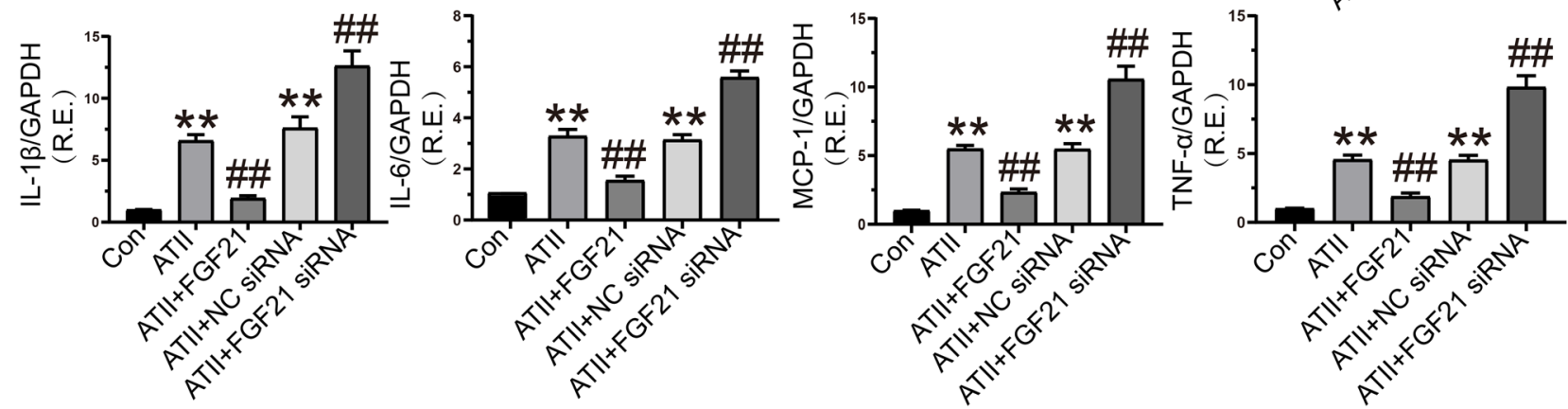

ROS production (Fig. 4d), relative migration index (Fig. 4e), and mRNA levels of proinflammatory cytokines including IL-1 $\beta$, IL-6, MCP-1, and TNF- $\alpha$ (Fig. 4f) in rat aortic AFs, which were further elevated by FGF21 siRNA but were largely decreased by recombinant FGF21. Therefore, our results suggested that FGF21 was a physiological protector against cellular damage and disorder in rat aortic AFs in response to ATII. 
4Fig. 4 Regulatory roles of FGF21 in cell apoptosis, oxidative stress, cell migration, and inflammatory response in rat aortic AFs. a Flow cytometry to detect cell apoptosis in rat aortic AFs. b, $\mathbf{c}$ Western blot analysis to examine the protein levels of bax and bcl-2. d, e ATIImediated the augmentation of oxidative stress and cell migration in rat aortic AFs were accelerated by FGF21 siRNA but was rescued by FGF21 stimulation. $\mathbf{f}$ The relative mRNA levels of proinflammatory cytokines in rat aortic AFs by RT-PCR. GAPDH was used as an endogenous control. $n=3-4$ for each group except for $\mathbf{f}$ where $n=5$. $* * P<0.01$ compared with the control group; $\# P<0.05$, \#\#P<0.01 compared with ATII or ATII + NC siRNA group. A.U. arbitrary units, R.E. relative expression, $A F s$ adventitial fibroblasts, $A T I I$ angiotensin II, $N C$ negative control, $F G F 21$ fibroblast growth factor $21, I L$ $1 \beta$ interleukin- $1 \beta, I L-6$ interleukin- $6, T N F-\alpha$ tumor necrosis factor- $\alpha$, $M C P-1$ monocyte chemoattractant protein-1

\section{ACE2 activation was responsible for the protective effects of FGF21 on ATII-induced cellular damage in rat aortic AFs}

To clarify the exact role and mechanism of FGF21, we explored whether ACE2 knockdown would affect FGF21mediated actions in rat aortic AFs. ATII-induced the loss of ACE2 in rat AFs was further reduced by the transfection of ACE2 siRNA and FGF21 siRNA, respectively (Fig. 5a, b, d), but was rescued by FGF21 stimulation (Fig. 5c, d), supporting that FGF21 improved the expression of ACE2 after ATII stimulation in rat aortic AFs. Furthermore, gene silencing of ACE2 by siRNA strikingly suppressed the inhibitory effects of FGF21 on ATII-induced the promotion of cell apoptosis (Fig. 5e, f), inflammatory injury (Fig. 5g), oxidative stress (Fig. 5h), and cell migration (Fig. 5i) in rat AFs. Collectively, our data implicated that ELA exerted its protective effects on ATII-induced vascular adventitial cell injury and dysfunction via the activation of FGF21-ACE2 signaling in rat aortic AFs (Fig. 6).

\section{Discussion}

Multiple adverse factors, including an imbalance in the proliferation and apoptosis, excessive oxidative stress products, and inflammatory cytokines, contribute to vascular adventitial damage and remodeling (Intengan and Schiffrin 2001; Wang et al. 2014a). The role of the adventitia in vascular remodeling and arterial hypertension has attracted increasing attention. The vascular adventitia is the principal "injurysensing tissue" of the blood vessel wall and exhibits the earliest and most prominent changes in vascular remodeling through the production of chemokines, cytokines, growth factors, and ROS (Intengan and Schiffrin 2001; Sartore et al. 2001). Adventitial remodeling involves anomalous proliferation and migration, oxidative stress, and inflammatory response of AFs, which are vital regulators of artery vascular wall structure and function when exposed to mechanical damage and pressure load (Sartore et al. 2001; Liu et al. 2015). Excessive adventitial remodeling causes early aortic maladaptation in ATII-induced hypertension (Bersi et al. 2016). The accumulating studies provide further evidence that AFs are major contributors to adventitial remodeling and result in the neointimal formation in vascular diseases. In this work, we demonstrate, for the first time, that ELA prevents ATII-mediated the promotion of cell migration, oxidative stress, apoptosis, and inflammatory injury in rat aortic AFs by activating the FGF21-ACE2 signaling.

The apelinergic system, including APJ and its two classes of endogenous ligands named ELA and apelin, has emerged as a key signaling pathway in regulating cardiovascular homeostasis and remodeling (Ma et al. 2020; Yang et al. 2017). ELA, a novel endogenous ligand for APJ, has been implicated to lower blood pressure, promote angiogenesis, and relax blood vessels to protect against cardiovascular dysfunction (Ma et al. 2020; Sato et al. 2017; Yang et al. 2017). Exogenous ELA infusion offers a potential therapy for hypertension by eliciting vasodilation via the suppression of intrarenal RAS. Additionally, ELA downregulates ACE expression and promotes ACE2 activity through regulating transcription factors Forkhead box M1 in stressed hearts (Sato et al. 2017). Circulating ELA levels are decreased in essential hypertensive individuals and the fall in endogenous ELA levels may be involved in the pathogenesis of hypertension-related vascular damage ( $\mathrm{Li}$ et al. 2020). The ELAAPJ axis exerts a powerful depressor effect by enhancing the ACE2/ACE ratio and the angiotensin (1-7)/ATII ratio (Ma et al. 2020; Murza et al. 2016; Song et al. 2020; Yang et al. 2017). In this work, we revealed that ATII stimulation contributed to marked decreases in the mRNA levels of ELA and ACE2 in rat aortic AFs, which were rescued by the replenishment of ELA. Conversely, the levels of ELA and ACE2 were further accelerated after ELA knockdown with siRNA in rat aortic AFs in response to ATII, indicating the mutual interaction among the RAS, ELA, and ACE2 signaling. Based on this result, we further evaluated the underlying mechanisms of ELA in protecting ATII-induced vascular cellular damage in rat aortic AFs. ATII-mediated pro-migratory, pro-oxidant, pro-apoptotic, and pro-inflammatory effects were largely abrogated with ELA stimulation but were further deteriorated by ELA siRNA, confirming the inhibitory roles of ELA in ATII-mediated actions in rat aortic AFs.

FGF21 is an endocrine hormone that regulates energy metabolism and insulin sensitivity and has been proposed to be a sensitive stress signal for maintaining metabolic homeostasis (Fisher and Maratos-Flier 2016). Emerging studies start to focus on the protective role of FGF21 in the cardiovascular system in vitro and in vivo (Domouzoglou et al. 2015). Genetic FGF21 deficiency significantly exacerbates ATII-induced hypertension and vascular dysfunction 

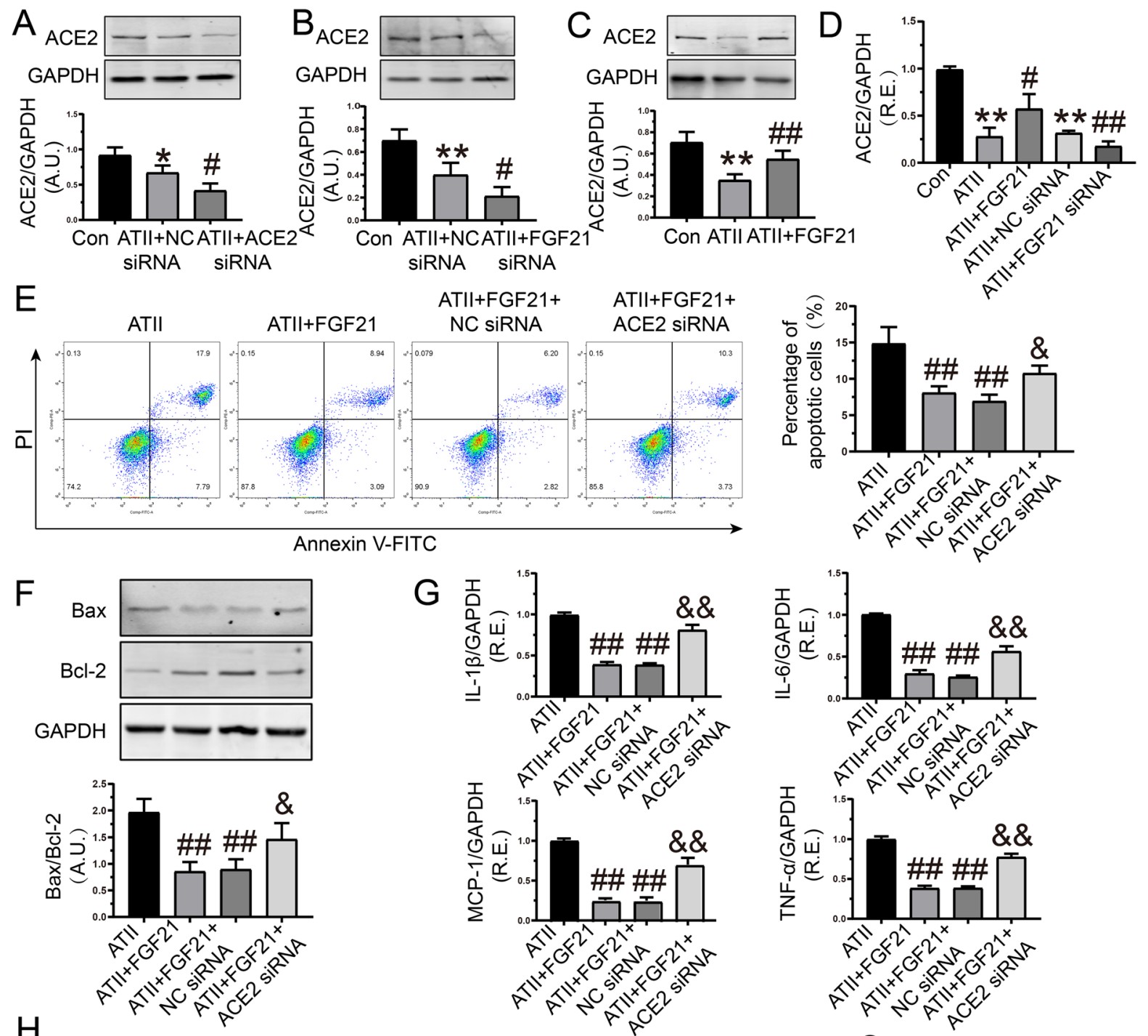

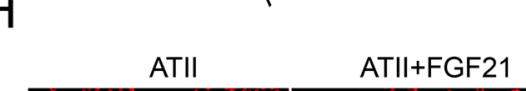

ATII+FGF21+ NC SiRNA

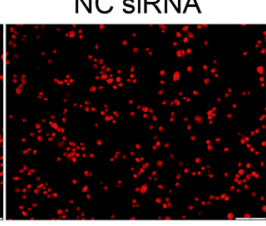

ATII+FGF21+

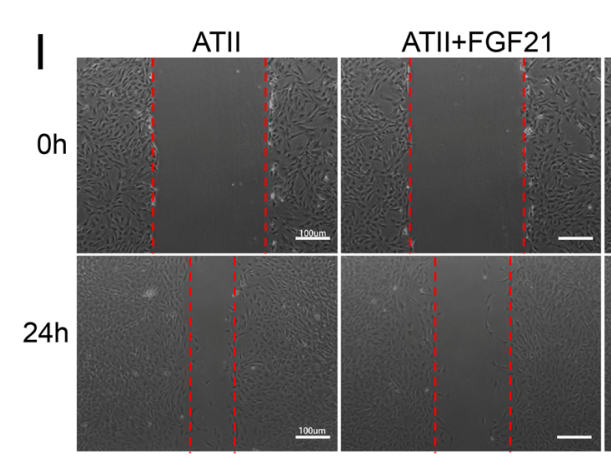

ATII+FGF21+ ACE2 siRNA

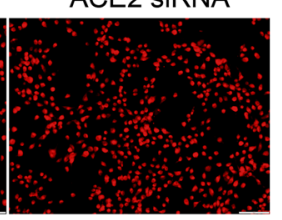

ATII+FGF21+ ACE2 SIRNA

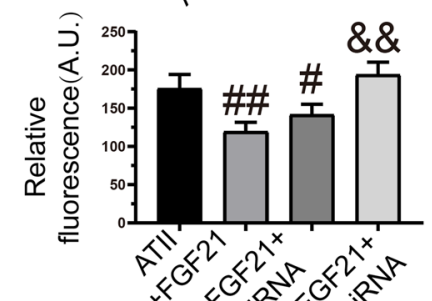

की

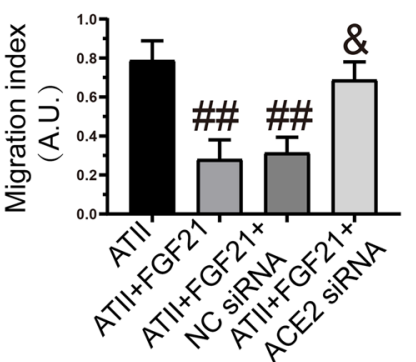


4Fig. 5 Protective roles of FGF21 in ATII-induced promotion of apoptosis, inflammatory response, oxidative stress, and cell migration were mediated by ACE2 signaling in rat aortic AFs. a The protein levels of ACE2 in the presence of ATII and ACE2 knockdown with siRNA. b-d Protein and mRNA levels of ACE2 in rat aortic AFs in response to ATII, FGF21, and FGF21 siRNA, respectively. e, f Cell apoptosis and apoptosis-associated proteins were detected by flow cytometry array and Western blots after exposure to ATII, FGF21, and ACE2 siRNA, respectively. $\mathbf{g}$ The expression of proinflammatory cytokines in rat aortic AFs by RT-PCR. h, i Dihydroethidium staining and wound healing assay to evaluate oxidative stress and cell migration in rat aortic AFs. GAPDH was used as an endogenous control. $\mathrm{n}=3-4$ for each group except for $\mathbf{d}$ and $\mathbf{g}$ where $\mathrm{n}=5-6$. $* P<0.05, * * P<0.01$ compared with the control group. $\# P<0.05$, $\# \# P<0.01$ compared with ATII or ATII + NC siRNA group. $\& \mathrm{P}<0.05, \& \& P<0.01$ compared with $\mathrm{ATII}+\mathrm{FGF} 21+\mathrm{NC}$ siRNA group; A.U. arbitrary units, R.E. relative expression, AFs adventitial fibroblasts, ATII angiotensin II, NC negative control, FGF21 fibroblast growth factor $21, A C E 2$ angiotensin-converting enzyme $2, I L$ $1 \beta$ interleukin- $1 \beta, I L-6$ interleukin-6, TNF- $\alpha$ tumor necrosis factor- $\alpha$, $M C P-1$ monocyte chemoattractant protein-1

and deteriorates diabetes-induced aortic thickening, fibrosis, inflammatory injury, and cell apoptosis (Yan et al. 2015; Planavila et al. 2013). Conversely, exogenous administration of FGF21 improves cardiac function and alleviates ATII-induced cardiac hypertrophy in a silent information regulator 1-dependent manner in mice, along with reduced ROS accumulation and cardiomyocyte apoptosis ( $\mathrm{Li}$ et al. 2019). Moreover, FGF21 attenuates aortic calcification by alleviating endoplasmic reticulum stress-mediated apoptosis in rats and prevents atherosclerosis in ApoE knockout mice by reducing vascular inflammation and apoptosis (Shi et al. 2019; Yan et al. 2018). FGF21 enhances angiogenesis and protects against high glucose-induced vascular damage and dysfunction in endothelial cells (Huang et al. 2019; Wang et al. 2014b). FGF21 prevents ATII-induced cerebrovascular aging in human brain vascular smooth muscle cells, which is related to the inhibition of ATII-induced ROS production levels (Wang et al. 2016). Recently, FGF21 was conformed to reduce blood pressure by regulating RAS signaling in a hypertensive rat model to exert beneficial effects on the process of hypertension and its related vascular damage. However, the role of FGF21 in vascular adventitial injury in hypertension has scarcely been investigated. In the present study, exposure to ATII induced a decline in the level of FGF21 in rat aortic AFs. Additionally, recombinant FGF21 protected vascular damage and dysfunction against ATIIinduced pro-oxidant, pro-apoptotic, pro-inflammatory, and pro-migratory actions, suggesting the antagonistic effect of FGF21 on ATII actions in rat aortic AFs. Moreover, the

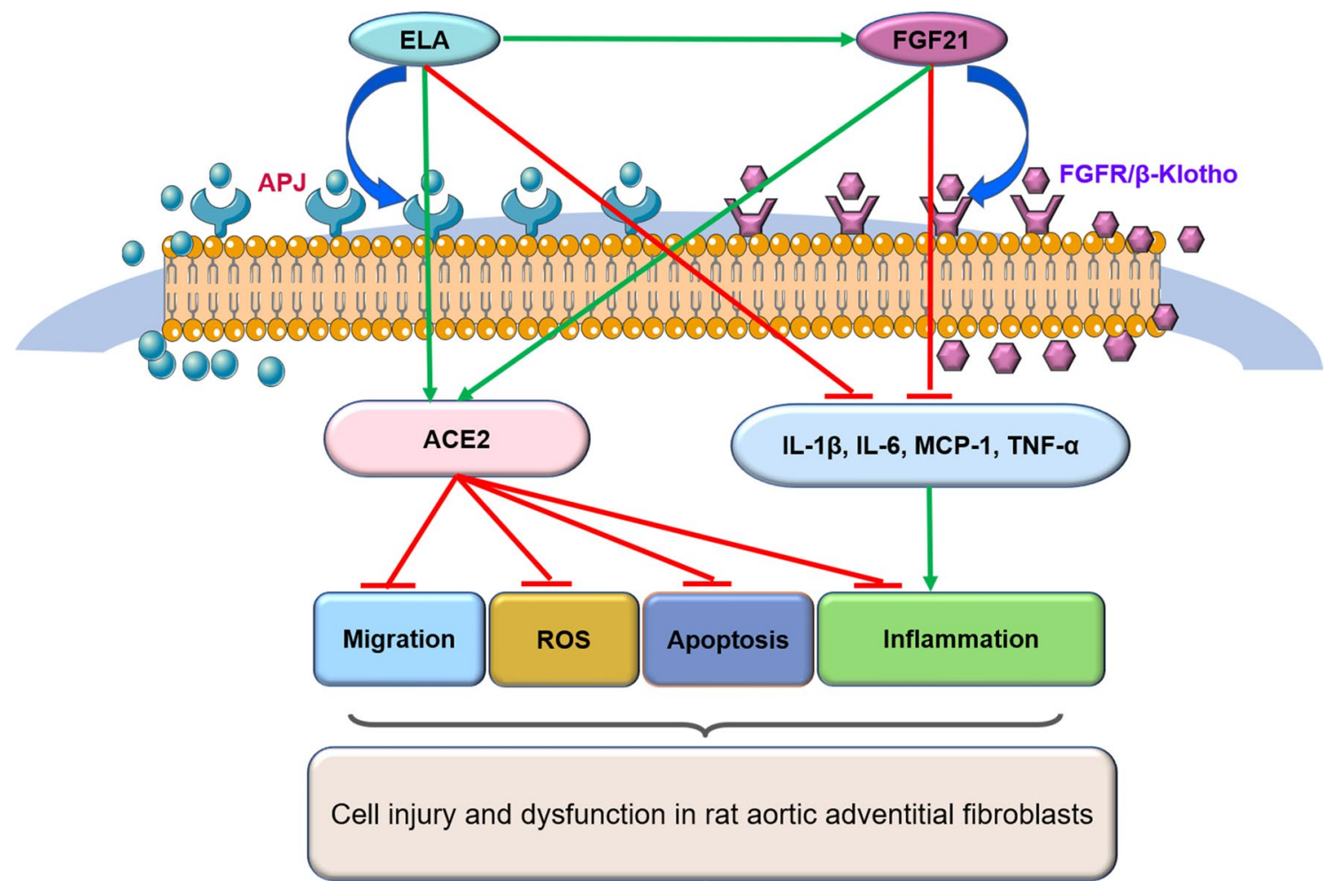

Fig. 6 Schematic overview of the crucial roles of Elabela in rat aortic adventitial fibroblasts. Elabela serves as a negative regulator of ATII-mediated the promotion of apoptosis, inflammatory injury, oxidative stress, and cell migration in rat aortic AFs by the activation of the FGF21-ACE2 signaling. ELA Elabela, FGF21 fibroblast growth factor 21, ACE2 angiotensin-converting enzyme 2, APJ G-protein coupled receptor, $F G F R$ fibroblast growth factor receptor, $R O S$ reactive oxygen species, $I L-1 \beta$ interleukin- $1 \beta, I L-6$ interleukin-6, TNF$\alpha$ tumor necrosis factor- $\alpha, M C P-1$ monocyte chemoattractant protein-1 
concentration of FGF21 in rat aortic AFs culture medium was reduced after exposure to ATII, which was partially rescued by exogenous supplement of ELA but was further decreased by the transfection of ELA siRNA. This reduction could be due to a compensatory response under the condition of ATII stimulation. Thus, it is likely that the reduced FGF21 level observed in rat aortic AFs in response to ATII is a compensatory mechanism, by which FGF21 is attempting to protect against vascular cellular injury and dysfunction in ATII-induced hypertension. Notably, the administration of ELA reversed ATII-mediated the loss of FGF21 level and gene silencing of FGF21 suppressed the anti-apoptotic, anti-oxidant, anti-migratory, and anti-inflammatory effects of ELA, indicating that the ELA-FGF21 signaling played an essential role in ATII-mediated vascular cellular injury regulation in rat aortic AFs.

Vasoconstriction, oxidative stress, mitochondrial dysfunction, inflammation, and fibrosis are some of the known mechanisms underlying in cardiovascular pathology induced by ACE/ATII/ATII type 1 receptor interaction. ACE2/Angiotensin (1-7)/Mas receptor axis has emerged as a physiological counter-regulatory peptide for the actions of ATII and is a critical protective pathway against cardiovascular, metabolic, and neurological disorders. Currently, increasing experimental studies regard the ACE2/Angiotensin (1-7)/ Mas axis as a potential therapeutic target for cardiovascular diseases (Gheblawi et al. 2020). Genetic ACE2 deletion results in the exacerbation of ATII-mediated cardiovascular fibrosis and oxidative stress in hypertensive mice, while the administration of recombinant human ACE2 remarkably rescues ATII-induced hypertension, pathological hypertrophy, oxidant injury, and cardiovascular remodeling (Sato et al. 2017; Zhong et al. 2010, 2011). Moreover, the activation of ACE2 signaling plays a key role in vascular repairment in chronic pathological conditions. Genetic ACE2 deficiency promotes vascular inflammatory response and atherosclerosis in the ApoE knockout mouse (Thomas et al. 2010). Conversely, ACE2 activation alleviates pulmonary vascular remodeling in rats with pulmonary hypertension (Yan et al. 2019), providing strong evidence for ACE2 to protect the vascular injury. The ELA-APJ axis protects from ATIIinduced cardiovascular damage and dysfunction in mice with transverse aorta constriction by enhancing the expression of ACE2 (Sato et al. 2017). Notably, FGF21 induces the increased activity of ACE2 derived from adipocytes and renal cells to protect against ATII-induced hypertension and vascular damage, suggesting that the FGF21-ACE2 signaling may serve as a novel therapeutic target in vascular injury and remodeling (Pan et al. 2018). In this study, we also demonstrated that the expression of ACE2 was significantly enhanced with ELA stimulation in rat AFs in response to ATII, whereas it was decreased by ELA siRNA treatment.
As expected, recombinant FGF21 improved the protein and mRNA levels of ACE2, which were decreased with FGF21 siRNA stimulation in rat AFs in response to ATII. Furthermore, the inhibitory effects of FGF21 on ATII-mediated the promotion of cell migration, oxidative stress, cell apoptosis, and inflammatory response were significantly prevented by the knockdown of ACE2, indicating that ACE2 is responsible for the protective roles of recombinant FGF21 in ATIIinduced vascular cellular damage in rat aortic AFs.

In conclusion, we uncover, for the first time, the protective effects of ELA on ATII-induced vascular cellular damage and dysfunction by suppressing the augmentation of cell migration, inflammatory injury, apoptosis, and oxidative stress in rat aortic AFs via the activation of the FGF21-ACE2 signaling. However, our study was performed in an experimental model, since there is a difference in hypertension susceptibility between humans and rodents, therefore more efforts should be made to better understand the therapeutic role of ELA against vascular damage and remodeling in hypertension in a humanoid large animal. Additionally, further prospective studies are needed to clarify whether ELA could be used as a prewarning biomarker and a novel therapeutic approach to identify the clinical risk of hypertension and hypertensive-related vascular injury and remodeling in individuals. Thus, an increased understanding of cardiovascular actions of the ELA-APJ axis would help to develop effective interventions for the prevention and treatment of hypertensive vascular injury. Targeting the FGF21-ACE2 signaling may be a promising therapeutic intervention for vascular adventitial remodeling and related disorders.

Acknowledgements This work was supported by the General Program and the National Major Research Plan Training Program of the National Natural Science Foundation of China (No. 81770253; 91849111; 81500038; 81300044; 81601660; 81870310; 81370362) and Talent Project of Beijing Chaoyang Hospital Affiliated to Capital Medical University.

Author contributions J-JS: Conceptualization, methodology, writing-original draft and revision, data analysis, making figures, writing - reviewing and editing; MY, YL, J-WS, and X-YL: Formal analysis, data curation, writing - original draft preparation; RM, Y-FF, and QZ: Visualization, investigation; YD and X-CY: Supervision, software, validation; Z-ZZ and YL: Methodology, data analysis, draw charts, and revision; J-CZ: Conceptualization, writing —original draft and revision, writing a review, and editing.

Funding This work was supported by the General Program and the National Major Research Plan Training Program of the National Natural Science Foundation of China (No. 81770253; 91849111; 81500038; $81300044 ; 81601660 ; 81870310 ; 81370362)$ and Talent Project of Beijing Chaoyang Hospital Affiliated to Capital Medical University.

Data availability The data used to support the findings of this study are available from the corresponding author upon request. 


\section{Declarations}

Conflict of interest All the authors declare that they have no conflict of interest.

Ethical approval Our study was approved by the Animal Research Ethics Committee at Beijing Chaoyang Hospital Affiliated to Capital Medical University.

\section{References}

Agarwal R (2003) Proinflammatory effects of oxidative stress in chronic kidney disease: role of additional angiotensin II blockade. Am J Physiol Renal Physiol 284(4):F863-F869. https://doi.org/ 10.1152/ajprenal.00385.2002

Bersi MR, Bellini C, Wu J, Montaniel KRC, Harrison DG, Humphrey JD (2016) Excessive adventitial remodeling leads to early aortic maladaptation in angiotensin-induced hypertension. Hypertension 67:890-896. DOI:https://doi.org/10.1161/HYPERTENSI ONAHA.115.06262

Chai X, Sun D, Han Q, Yi L, Wu Y, Liu X (2018) Hypoxia induces pulmonary arterial fibroblast proliferation, migration, differentiation and vascular remodeling via the PI3K/Akt/p70S6K signaling pathways. Int J Mol Med 41:2461-2472. DOI:https://doi.org/10. 3892/ijmm.2018.3462

Domouzoglou EM, Naka KK, Vlahos AP, Papafaklis MI, Michalis LK, Tsatsoulis A, Marators-Flier E (2015) Fibroblast growth factors in cardiovascular disease: the emerging role of FGF21. Am J Physiol Heart Circ Physiol 309:H1029-H1038. DOI:https://doi. org/10.1152/ajpheart.00527.2015

Fisher FM, Maratos-Flier E (2016) Understanding the physiology of FGF21. Annu Rev Physiol 78:223-241. https://doi.org/10.1146/ annurev-physiol-021115-105339

Gheblawi M, Wang K, Viveiros A, Nguyen O, Zhong J, Turner AJ, Raizada MK, Grant MB, Oudit GY (2020) Angiotensin converting enzyme 2: SARS-CoV-2 receptor and regulator of the reninangiotensin system - celebrating the 20th Anniversary of the Discovery of ACE2. Circ Res 126:1457-1475. https://doi.org/ 10.1161/CIRCRESAHA.120.317015

Helker CS, Schuermann A, Pollmann C, Chng SC, Kiefer F, Reversade B, Herzog W (2015) The hormonal peptide elabela guides angioblasts to the midline during vasculogenesis. Elife 4:1-13. https:// doi.org/10.7554/eLife.06726

Huang W, Shao M, Liu H, Chen J, Hu J, Zhu L, Liu F, Wang D, Zou Y, Xiong Y, Wang X (2019) Fibroblast growth factor 21 enhances angiogenesis and wound healing of human brain microvascular endothelial cells by activating PPAR $\gamma$. J Pharmacol Sci 140:120127. DOI:https://doi.org/10.1016/j.jphs.2019.03.010

Intengan HD, Schiffrin EL (2001) Vascular remodeling in hypertension: roles of apoptosis, inflammation, and fibrosis. Hypertension 38:581-587. DOI:https://doi.org/10.1161/hy09t1.096249

Li G, Zhang H, Zhao L, Zhang Y, Yan D, Liu Y (2017) Angiotensinconverting enzyme 2 activation ameliorates pulmonary endothelial dysfunction in rats with pulmonary arterial hypertension through mediating phosphorylation of endothelial nitric oxide synthase. J Am Soc Hypertens 11:842-852. https://doi.org/10. 1016/j.jash.2017.10.009

Li Y, Yang X, Ouyang S, He J, Yu B, Lin X, Zhang Q, Tao J (2020) Declined circulating Elabela levels in patients with essential hypertension and its association with impaired vascular function: a preliminary study. Clin Exp Hypertens 42:239-243. https://doi. org/10.1080/10641963.2019.1619756
Li S, Zhu Z, Xue M, Yi X, Liang J, Niu C, Chen G, Shen Y, Zhang H, Zheng J, Zhao C, Liang Y, Cong W, Wang Y, Jin L (2019) Fibroblast growth factor 21 protects the heart from angiotensin II-induced cardiac hypertrophy and dysfunction via SIRT1. BBAMol Basis Dis 1865:1241-1252. https://doi.org/10.1016/j.bbadis. 2019.01.019

Lin Z, Pan X, Wu F, Ye D, Zhang Y, Wang Y, Jin L, Lian Q, Huang Y, Ding H, Triggle C, Wang K, Li X, Xu A (2015) Fibroblast growth factor 21 prevents atherosclerosis by suppression of hepatic sterol regulatory element-binding protein- 2 and induction of adiponectin in mice. Circulation 131:1861-1871. DOI:https://doi.org/10.1161/ CIRCULATIONAHA.115.015308

Liu CF, Zhang J, Shen K, Gao PJ, Wang HY, Jin X, Meng C, Fang NY (2015) Adventitial gene transfer of catalase attenuates angiotensin II-induced vascular remodeling. Mol Med Rep 11:2608-2614. DOI:https://doi.org/10.3892/mmr.2014.3069

Luo Y, Lu W, Li X (2018) Unraveling endocrine FGF signaling complex to combat metabolic diseases. Trends Biochem Sci 43:563566. https://doi.org/10.1016/j.tibs.2018.05.001

Ma Z, Song JJ, Martin S, Yang XC, Zhong JC (2020) The Elabela-APJ axis: a promising therapeutic target for heart failure. Heart Fail Rev 26:1-10. https://doi.org/10.1007/s10741-020-09957-5

Murza A, Sainsily X, Coquerel D, Cote J, Marx P, Besserer-Offroy E, Longpre JM, Laine J, Reversade B, Salvail D, Leduc R, Dumaine R, Lesur O, Auger-Messier M, Sarret P, Masault E (2016) Discovery and structure-activity relationship of a bioactive fragment of ELABELA that modulates vascular and cardiac functions. J Med Chem 59:2962-2972. https://doi.org/10.1021/acs.jmedchem. $5 \mathrm{~b} 01549$

Pan X, Shao Y, Wu F, Wang Y, Xiong R, Zheng J, Tian H, Wang B, Wang Y, Zhang Y, Han Z, Qu A, Xu H, Lu A, Yang T, Li X, Xu A, Du J, Lin Z (2018) FGF21 prevents angiotensin ii-induced hypertension and vascular dysfunction by activation of ACE2/ angiotensin-(1-7) axis in mice. Cell Metab 27:1323-1337. https:// doi.org/10.1016/j.cmet.2018.04.002

Planavila A, Redondo I, Hondares E, Vinciguerra M, Munts C, Iglesias R, Gabrirlli LA, Sitges M, Giralt M, van Bilsen M, Villarroya F (2013) Fibroblast growth factor 21 protects against cardiac hypertrophy in mice. Nat Commun 4:2019. https://doi.org/10.1038/ ncomms3019

Sartore S, Chiavegato A, Faggin E, Franch R, Puato M, Ausoni S, Pauletto P (2001) Contribution of adventitial fibroblasts to neointima formation and vascular remodeling: from innocent bystander to active participant. Circ Res 89:1111-1121. DOI:https://doi.org/ 10.1161/hh2401.100844

Sato T, Sato C, Kadowaki A, Watanabe H, Ho L, Ishida J, Yamaguchi T, Kimura A, Penninger JM, Reversade B, Ito H, Imai Y, Kuba K (2017) ELABELA-APJ axis protects from pressure overload heart failure and angiotensin II-induced cardiac damage. Cardiovasc Res 113:760-769. DOI:https://doi.org/10.1093/cvr/cvx061

Shi Y, Wang S, Peng H, Lv Y, Li W, Cheng S, Liu J (2019) Fibroblast growth factor 21 attenuates vascular calcification by alleviating endoplasmic reticulum stress mediated apoptosis in rats. Int J Biol Sci 15:138-147. https://doi.org/10.7150/ijbs.28873

Song JJ, Yang M, Liu Y, Song JW, Wang J, Chi HJ, Liu XY, Zuo K, Yang XC, Zhong JC (2020) MicroRNA-122 aggravates angiotensin II-mediated apoptosis and autophagy imbalance in rat aortic adventitial fibroblasts via the modulation of SIRT6-elabela-ACE2 signaling. Eur J Pharmacol 883:173374. DOI:https://doi.org/10. 1016/j.ejphar.2020.173374

Thomas MC, Pickering RJ, Tsorotes D, Koitka A, Sheehy K, Bernardi S, Toffoli B, Nguyen-Huu TP, Head GA, Fu Y, Chin-Dusting J, Cooper ME, Tikellis C (2010) Genetic Ace2 deficiency accentuates vascular inflammation and atherosclerosis in the ApoE knockout mouse. Circ Res 107:888-897. https://doi.org/ 10.1161/CIRCRESAHA.110.219279 
Wang XM, Song SS, Xiao H, Gao P, Li XJ, Si LY (2014b) Fibroblast growth factor 21 protects against high glucose induced cellular damage and dysfunction of endothelial nitric-oxide synthase in endothelial cells. Cell Physiol Biochem 34:658-671. DOI:https://doi.org/10.1159/000363031

Wang XM, Xiao H, Liu LL, Cheng D, Li XJ, Si LY (2016) FGF21 represses cerebrovascular aging via improving mitochondrial biogenesis and inhibiting p53 signaling pathway in an AMPKdependent manner. Exp Cell Res 346:147-156. DOI:https://doi. org/10.1016/j.yexcr.2016.06.020

Wang Z, Yu D, Wang M, Wang Q, Kouznetsova J, Yang R, Qian K, Wu W, Shuldiner A, Sztalryd C, Zou M, Zheng W, Gong DW (2015) Elabela-apelin receptor signaling pathway Is functional in mammalian systems. Sci Rep 5:8170. https://doi.org/10.1038/ srep08170

Wang D, Zhang H, Li M, Frid MG, Flockton AR, McKeon BA, Yeager ME, Fini MA, Morrell NW, Pullamsetti SS, Velegala S, Seeger W, McKinsey TA, Sucharov CC, Stenmark KR (2014a) MicroRNA-124 controls the proliferative, migratory, and inflammatory phenotype of pulmonary vascular fibroblasts. Circ Res 114:67-78. DOI:https://doi.org/10.1161/CIRCRESAHA.114.301633

Williams VR, Scholey JW (2018) Angiotensin-converting enzyme 2 and renal disease. Current Opinion Nephrology Hypertension 27:35-41. DOI:https://doi.org/10.1097/MNH.0000000000000378

Xu R, Zhang ZZ, Chen LJ, Yu HM, Guo SJ, Xu YL, Oudit GY, Zhang Y, Chang Q, Song B, Chen DR, Zhu DL, Zhong JC (2016) Ascending aortic adventitial remodeling and fibrosis are ameliorated with Apelin-13 in rats after TAC via suppression of the miRNA-122 and LGR4- $\beta$-catenin Signaling. Peptides 86:85-94. DOI:https://doi.org/10.1016/j.peptides.2016.10.005

Yan X, Chen J, Zhang C, Zeng J, Zhou S, Zhang Z, Lu X, Chen J, Feng W, Li X, Tan Y (2015) Fibroblast growth factor 21 deletion aggravates diabetes-induced pathogenic changes in the aorta in type 1 diabetic mice. Cardiovasc Diabetol 14:77. DOI:https://doi. org/10.1186/s12933-015-0241-0

Yan X, Gou Z, Li Y, Wang Y, Zhu J, Xu G, Zhang Q (2018) Fibroblast growth factor 21 inhibits atherosclerosis in ApoE-/- mice by ameliorating Fas-mediated apoptosis. Lipids Health Dis 17:203. DOI:https://doi.org/10.1186/s12944-018-0846-x
Yan D, Li G, Zhang Y, Liu Y (2019) Angiotensin-converting enzyme 2 activation suppresses pulmonary vascular remodeling by inducing apoptosis through the Hippo signaling pathway in rats with pulmonary arterial hypertension. Clin Exp Hypertens 41:589-598. DOI:https://doi.org/10.1080/10641963.2019.1583247

Yang P, Read C, Kuc RE, Buonincontri G, Southwood M, Torella R, Upton PD, Crosby A, Sawiak SJ, Carpenter TA, Glen RC, Morrell NW, Maguire JJ, Davenport AP (2017) Elabela/toddler is an endogenous agonist of the apelin APJ receptor in the adult cardiovascular system, and exogenous administration of the peptide compensates for the downregulation of its expression in pulmonary arterial hypertension. Circulation 135:1160-1173. DOI:https://doi.org/10.1161/CIRCULATIONAHA.116.023218

Ying L, Li N, He Z, Zeng X, Nan Y, Chen J, Miao P, Ying Y, Lin W, Zhao X, Lu L, Chen M, Cen W, Guo T, Li X, Huang Z, Wang Y (2019) Fibroblast growth factor 21 ameliorates diabetes induced endothelial dysfunction in mouse aorta via activation of the CaMKK2/AMPK $\alpha$ signaling pathway. Cell Death Dis 10:665. https://doi.org/10.1038/s41419-019-1893-6

Zhong J, Basu R, Guo D, Chow FL, Byrns S, Schuster M, Loibner H, Wang XH, Penninger JM, Kassiri Z, Oudit GY (2010) Angiotensin-converting enzyme 2 suppresses pathological hypertrophy, myocardial fibrosis and cardiac dysfunction. Circulation 122:717-728. DOI:https://doi.org/10.1161/CIRCULATIONAHA. 110.955369

Zhong J, Guo D, Chen CB, Wang W, Schuster M, Loibner H, Penninger JM, Scholey JW, Kassiri Z, Oudit GY (2011) Prevention of angiotensin II-mediated renal oxidative stress, inflammation, and fibrosis by angiotensin-converting enzyme 2. Hypertension 57:314-322. https://doi.org/10.1161/HYPERTENSIONAHA.110. 164244

Publisher's Note Springer Nature remains neutral with regard to jurisdictional claims in published maps and institutional affiliations. 